\section{BS21 CHARACTERISATION OF AN IN VITRO MODEL FOR THE DONATION OF PHYSIOLOGICAL INORGANIC NITRATE FROM DIETARY SOURCES: PHARMACOKINETICS AND PHARMACODYNAMICS}

${ }^{1}$ Genna Logue*, ${ }^{2}$ Fairoz Belary Abdul. ${ }^{1}$ Wales Heart Research Institute; ${ }^{2}$ University Hospital of Wales

10.1136/heartjn--2019-BCS.184

Background Nitric oxide (NO) has anti-platelet activity. ${ }^{1}$ Nitrate, nitrite and nitrosthiols (SNO) are $\mathrm{NO}$ intermediates. ${ }^{2}$ Exogenous NO sources include dietary inorganic nitrate and commercial products, Beet It (BI) juice and SIS ${ }^{\circledR}$ Go + nitrate (SGN) gel. $^{3}$ In the entero-salivary circulation nitrate reduces to nitrite via nitrate reductase (NR) from commensal oral bacteria. ${ }^{4}$ Nitrite reacts with gastric proteins to form R-SNO; clopidogrel may enhance production. ${ }^{5}$ Patients with coronary artery disease (CAD) have impaired endogenous NO generation. ${ }^{67}$

Methods A model was created enabling in vitro sodium nitrate salt (SNS) reduction by NR from Aspergillus niger. ${ }^{8}$ SGN gel, placebo gel and BI were tested using this. Product was added to gastric medium to form R-SNO, with and without clopidogrel. ${ }^{5}$ NO metabolites were quantified using ozone-based chemiluminescence. ${ }^{9}$

Results SGN gel and BI produced limited nitrite. Placebo gel (nitrate deficient) mixed with SNS also produced limited nitrite. Gel dilution improved yield. R-SNO production was greater from nitrite converted from SGN gel than SNS; clopidogrel did not enhance yield.

Key messages A model was established for the reduction of nitrate from commercial agents. R-SNO formed in gastric medium in the presence of nitrite. This helps understand a clinical study assessing therapeutic effects of nitrate on platelet activity in CAD. ${ }^{1}$

\section{REFERENCES}

1. Halliwel B. Free Radicals and other reactive species in Disease. Encyclopedia of life Sciences 2003:1-7.

2. Kim-Shapiro DB, Schechter AN, Gladwin MT. Unraveling the Reactions of Nitric Oxide, Nitrite, and Hemoglobin in Physiology and Therapeutics. Arteriosclerosis, Thrombosis, and Vascular Biology 2006;26:697-705.

3. Cermak NM, Gibala MJ, vanLoon LJC. Nitrate Supplementation's Improvement of 10-km Time-Trial Performance in Trained Cyclists. International Journal of Sport Nutrition and Exercise Metabolism 2012;22:64-71.

4. O. Lundberg J, Carlstrom M, Larsen FL, Weitzberg. Roles of dietary inorganic nitrate in cardiovascular health and disease. Cardiovascular Research 2011:89:525-532.

5. Bundhoo SS, Anderson RA, Sagan E, Hassan N, Pinder AG, Rogers SC, et al. Direct formation of thienopyridine-derived nitrosothiols-just add nitrite! European Journal of Pharmacology 2011 November;670(2-3):534-40.

6. Baliga RS, Milsom AB, Ghosh SS, Trinder SL, MacAllister RJ, Ahluwalia A, et al. Dietary nitrate ameliorates pulmonary hypertension: cytoprotective role for endothelial nitric oxide synthase and xanthine oxidoreductase. Circulation. American Heart Association 2012;125:2922-2932.

7. Webb AJ, Patel N, Loukogeorgakis S, Okorie M, Aboud Z, Misra S, et al. Nitric oxide, Oxidative stress. Acute blood pressure lowering, vasoprotective, and antiplatelet properties of dietary nitrate via bioconversion to nitrite. Hypertension. American Heart Association 2008;51:784-790.

8. Milsom AB. An assessment of Nitric Oxide metabolism in blood: a physiological role for Nitric Oxide metabolites and the implications for diabetes mellitus [PhD thesis]. Cardiff: Cardiff University; 2003.

9. Burnley-Hall N, Abdul F, Androshchuk V, Morris K, Ossei-Gerning N, Anderson R, Rees A, James P. Dietary Nitrate Supplementation Reduces Circulating PlateletDerived Extracellular Vesicles in Coronary Artery Disease Patients on Clopidogrel Therapy: A Randomised, Double-Blind, Placebo-Controlled Study. Thrombosis and Haemostasis 2018;118:112-122.

\section{BS22 DOUBLE POSITIVE (CD86+ MRC1+) INFLAMMATORY MACROPHAGES IN THE PATHOGENESIS OF CAROTID ATHEROSCLEROSIS}

${ }^{1}$ Klaudia Kocsy*, ${ }^{2}$ Sheila Francis, ${ }^{3}$ Endre Kiss-Toth, ${ }^{4}$ Robert Lonsdale, ${ }^{5}$ Arshad Majid, ${ }^{5}$ Jessica Redgrave. 'Department of Infection, Immunity \& Cardiovascular Disease, University of Sheffield; ${ }^{2}$ University of Sheffield; ${ }^{3}$ Department of Infection, Immunity \& Cardiovascular Disease; ${ }^{4}$ Sheffield Teaching Hospitals NHS Foundation Trust; ${ }^{5}$ Sheffield Institute for Translational Neuroscience

\subsection{6/heartjnl-2019-BCS.185}

Introduction Atherosclerosis is a complex inflammatory disease in which major arteries narrow, and an atherosclerotic plaque develops, modulated by genetic and environmental factors. Macrophages participate in all stages of the plaque formation and progression. Different macrophage subtypes within carotid plaques have been shown to be altered in unstable vs. stable plaques. M1 proinflammatory macrophages are mostly abundant in symptomatic and unstable plaques. In contrast, M2 macrophages are associated with regulatory and wound-healing properties and generally found in stable lesions. In the future, the characterisation of these changes in situ in the carotid plaques, associated with in vitro macrophage studies, and blood composition may lead to the identification of diagnostic markers to direct the best treatment for stroke patients.

Methods Carotid plaques were obtained from patients with a recent stroke or TIA and with a greater than 50\% stenosis of the internal carotid artery. Plaques were removed during carotid endarterectomy and fixed in $10 \%(\mathrm{v} / \mathrm{v})$ neutral buffered formalin for maximum 36h. Macrophage phenotypes within the shoulder regions were characterised with immunofluorescence microscopy. A novel immunofluorescence staining, image acquisition and analysis technique was developed to identify single M1 (CD68+CD86+MRC1-), single M2 (CD68+CD86$\mathrm{MRC} 1+)$, double positive $(\mathrm{CD} 68+\mathrm{CD} 86+\mathrm{MRC} 1+)$ and double negative (CD68+CD86-MRC1-) macrophages in the human carotid atherosclerotic plaques.

Results 20 carotid plaques were collected from patients with recent stroke or TIA (73\% male, $70 \%$ smokers, $87 \%$ hypertensive). In 17 plaques the shoulder region (either side of a thinned fibrous cap) was sufficiently intact for histological analysis. Dense infiltration with macrophages was seen in all 17 specimens. The population comprised double positive (CD86+MRC1+) (71\%), M1 single positive (CD86+MRC1-) (19\%), and M2 single positive (CD86-MRC1+) (4\%). Double negative (CD86-MRC1-) macrophages represented 6\% of all macrophages. Amongst 10 unstable plaques (defined as AHA Grade 6 or above) the majority (80\%) of shoulder macrophages were M1 single positive. In contrast, amongst 7 stable plaques, the majority of plaque shoulder macrophages $(65 \%)$ were M2 single positive.

Conclusions Macrophages within symptomatic carotid plaques can express both M1 (CD86) and M2 (MRC1) markers, or can express neither. Pro-inflammatory macrophage phenotypes cluster in areas of unstable carotid plaque. Larger studies are needed to confirm the role of dual staining macrophages and double negative macrophages. If such in situ macrophages can be manipulated towards one phenotype with pharmaceutical interventions, this could offer a potential new approach to plaque stabilising therapies.

Conflict of interest No 